\title{
Why granular media are thermal after all
}

\author{
Mario Liu ${ }^{1, \star}$ and Yimin Jiang ${ }^{2}$ \\ ${ }^{1}$ Theoretische Physik, Universität Tübingen,72076 Tübingen, Germany \\ ${ }^{2}$ Central South University, Changsha 410083, China
}

\begin{abstract}
Two approaches exist to account for granular behavior. The thermal one considers the total entropy, which includes microscopic degrees of freedom such as phonons; the athermal one (as with the Edward entropy) takes grains as elementary. Granular solid hydrodynamics (GSH) belongs to the first, DEM, granular kinetic theory and athermal statistical mechanics (ASM) to the second. A careful discussion of their conceptual differences is given here. Three noteworthy insights or results are: (1) While DEM and granular kinetic theory are well justified to take grains as elementary, any athermal entropic consideration is bound to run into trouble. (2) Many general principles are taken as invalid in granular media. Yet within the thermal approach, energy conservation and fluctuation-dissipation theorem remain valid, granular temperatures equilibrate, and phase space is well explored in a grain at rest. Hence these are abnormalities of the athermal approximation, not of granular media as such. (3) GSH is a wide-ranged continuum mechanical description of granular dynamics.
\end{abstract}

\section{Introduction}

Taking grains as elementary particles interacting via the Newtonian law, the discrete element method (DEM) is the tool of choice coming to terms with granular behavior. Similarly, granular kinetic theory also assumes that grains are structureless particles undergoing dissipative collisions. Both results have contributed to the widespread believe in the physics community that grains may generally be approximated as elementary. Starting from this believe, "athermal statistical mechanics" (ASM) defines a reduced entropy $S_{g}$ that does not contain any microscopic degrees of freedom, only those of granular configurations, and assumes it is maximal in equilibrium [1]. One example is the Edward entropy, $S_{E d}$, given by the number of possibilities grains may be stably packed $[2,3]$.

This is a leap of faith. The irrelevance of microscopics for DEM or the kinetic theory does not imply that the true entropy $S$, of all the microscopic degrees of freedom such as phonons and free electrons, is irrelevant. Brownian motion is negligible because grains are macroscopically large, each containing many, many microscopic degrees of freedom, such that $S \gg S_{g}$. Taking $S+S_{g}$ to be maximal in equilibrium implies that equilibrium holds as long as $S$ is maximal, pretty much irrespective of $S_{g}$.

The lack of Brownian motion shows that phonons and electrons do not move grains. But this is quite insufficient for the conclusion of athermality. Crucial is the question: Which of the two entropies, $S_{g}$ or $S$, is being increased by dissipation off equilibrium, during collisions and relaxations, and becomes maximal in equilibrium? Grains are athermal only if $S_{g}$ is the answer. However, whenever a

\footnotetext{
^e-mail:mliu@uni-tuebingen.de
}

system loses energy and heats up measurably - by fingers or a thermometer, it is $S$ that is being increased. This is what grains do in experiments, and - if one would trace the lost energy - also in DEM. And this is why GSH takes $S$ to be maximal in equilibrium, with dissipative terms constructed to increase $S$ off-equilibrium. ASM takes $S_{g}$ to be maximal, and as yet shuns discussion of dissipation.

Note that since neither DEM nor the kinetic theory deal with the entropy, their obvious usefulness in accounting for experiments does not support the ASM assumption. What is more, both possess the appropriate dissipative terms to reach the proper equilibrium, with all grains at rest, given by $S=\max$. Note also that GSH successfully describes a wide range of granular phenomena [4], including fast dense flow, elaso-plastic motion, static stress distribution, propagation of elastic waves, and compaction. And it reduces, in the respective limits, to hypoplasticity [5], Kamrin's nonlocal constitutive relation [6], the $\mu(I)$-rheology, and the hydrodynamic equations of granular gases. GSH also contains mathematical structures of striking similarity to glassy dynamics, including critical slowing down, aging and memory. Given these results, GSH appears well-positioned to present a unified macroscopic framework for coming to terms with the multitude of granular phenomena.

As yet, GSH is a qualitative theory, providing a bird's eye view of granular behavior. But we are striving to make it quantitative. Its input in basic understanding are twostage irreversibility and variable transient elasticity. The first addresses the three length scales of any granular media-macroscopic, granular and microscopic, and the fact that energy in the macroscopic degrees of freedom typically first decays into the granular, then the microscopic 
ones. The second addresses the fact that the elastic stress relaxes when grains jiggle, and that it relaxes faster the stronger the jiggling is.

These two concepts introduce the granular temperature $T_{g}$ (conjugate to $S_{g}$ ) and the elastic strain $u_{i j}$ as additional state variables. Hereby, $u_{i j}$ accounts for the coarse-grained elastic deformation of the grains, and the associated elastic energy, while $T_{g}$ quantifies the quickly fluctuating elastic and kinetic energy of individual grains. Both obey relaxation equations, pushing the system toward equilibrium, $S=\max$, and accounting for granular dynamics on their way there. This is the most ostentatious link between microscopics (phonons and electrons) and macroscopics (granular flow and stacking). In addition, GSH contains the conservation equations for mass, momentum and energy, and a balance equation for $S$.

\section{Two models for grains}

There are two models for grains, a gas of particles taken as elementary, and a block of thermal rock that has been sliced and diced. The first is athermal, the second thermal. Both work well within their respective range of validity, but one needs to know what they are. The simple example of a pendulum is helpful here.

\subsection{Is a pendulum athermal?}

A pendulum is, like a grain, a macroscopic object. The equation, $\ddot{\theta}+\alpha \dot{\theta}+\theta g / l=0$, accounts for its small-amplitude motion, with no need to consider its entropy $S$. ( $\theta$ : pendulum angle, $g$ : gravitational constant, $l$ length of the string, $\alpha$ : friction coefficient.) Still, a pendulum is not athermal, because the frictional force $\alpha \dot{\theta}$ increases $S$.

This force is derived by starting from the second law of thermodynamics, ie. $S$ only increases, until it is maximal in equilibrium: The conserved energy $E$ is given by the potential, kinetic and heat contributions, $E=\frac{1}{2} M g l \theta^{2}+$ $\frac{1}{2} M l^{2} \dot{\theta}^{2}+\int T \mathrm{~d} S$, or $\dot{E}=0=M g l \dot{\theta}+M l^{2} \dot{\theta} \ddot{\theta}+T \dot{S}$. Inserting the pendulum equation, $\ddot{\theta}+Y+\theta g / l=0$, with $Y$ unknown, we find $\dot{S}=\left(M l^{2} / T\right) \dot{\theta} Y>0$. Since $M l^{2} / T>0$, but not necessarily $\dot{\theta}$, we set $Y=\alpha \dot{\theta}, \alpha>0$. This force acts until $\theta, \dot{\theta}=0$, implying $E=\int T \mathrm{~d} S$, or $S=\max$.

Clearly, a pendulum is both athermal and thermal athermal when the dissipative force is known, thermal if it needs to be derived. Grains are not different. Because DEM and granular kinetic theory possess the proper dissipative forces, they may treat grains as elementary. But if one needs to derive the dissipative terms of a macroscopic theory, it is not clear how one can possibly avoid $S$.

\subsection{Athermal gas and thermal solid}

Faced with the task to setup a macroscopic, continuummechanical theory, it may seem natural to model grains as a gas of elementary particles, because it seems hard to imagine how phonons inside the grains could possibly influence their flow and stacking. However, see the last paragraph of the introduction. Moreover, athermal grains and gaseous molecules are far apart, mainly because energy conservation does not hold for grains, hence statistical mechanics does not work. ASM tries to ameliorate this by replacing the energy with volume or stress. Yet energy conservation, related to time translational symmetry, is a fundamental property of matter; constancy of volume or stress are experimental prescriptions.

There are further problems: Failure of granular temperatures to equilibrate, invalidity of the fluctuationdissipation theorem, jammed grains lack the possibility to explore the phase space... All these in addition to the basic problem, $S \gg S_{g}$. If the gas model poses such difficulties, the question whether a solid is better suited as a starting point, to develop a macroscopic theory for granular media, naturally arises. The answer: grains are often well modeled by a thermal rock that has been sliced and diced.

Modelling a block of solid including the gas of quasiparticles such as phonons and free electrons, the energy dissipating into them is not lost. With the energy conserved, thermodynamics holds, and statistical mechanics works. Crucially, same is true for a stack of two blocks, or a pile of grains - as long as they are macroscopic.

The notion that grains at rest are jammed, in need of shaking for phase space exploration, is now inappropriate: Phonons roam nearly as freely in a pile of grains as in a block of rock. The fluctuation-dissipation theorem, dealing with thermal fluctuations of phonons and electrons, also holds in granular media. Finally, that granular temperatures $T_{g}$ do not equilibrate is traceable to the neglect of the true temperature $T$ : Given two systems, each with a granular and a true temperature, the behavior is completely analogous to four systems with four temperatures. There is little unique or incomprehensible about $T_{g}$ 's behavior.

With the microscopics reassuringly healthy, we proceed to consider the macroscopic description of granular dynamics. A block of solid is an elastic medium. Cutting the block in half, with one part on top of the other, we expect them to remain elastic under shear if they do not slip. If they do, we subtract the slipping portion from the total displacement to obtain the deforming one. Further slicing the block to eventually arrive at many (macroscopic) pieces, the system is still elastic - as long as we keep track of the deforming displacement. We call the associated strain field elastic, coarse-grain it, and denote it as $u_{i j}$. Since the elastic stress $\pi_{i j}$ stems from deformed grains, it is a function of $u_{i j}$, and of the density, $\pi_{i j}=\pi_{i j}\left(\rho, u_{k l}\right)$.

Because force equilibrium, $\nabla_{j} \pi_{i j}=\rho g_{i}$, is the condition for $S=\max$ with respect to variations of $u_{i j}$ [7-9], any stable stacking of grains (the ones being counted in the Edward entropy), is an equilibrium configuration.

To see how misleading the reduced entropy $S_{g}$ may be, consider an ensemble of pendulums in equilibrium all hanging down, no deviations allowed. Since there is only this one state, we have $S_{g}=0$ being minimal. And the reason is: A pendulum coming to rest has maximal $S$.

Grains are of course more complicated than noninteracting pendulums. Jamming and obstructing one another, they have $S_{g} \neq 0$, and give rise to a panoply of phenomena. Nevertheless, taking the total entropy, $S_{t o t}=$ 
$S+S_{g}$, from all available degrees of freedom, to be maximal in equilibrium is always correct. With $S_{g} \ll S$, taking $S=\max$ is an excellent approximation. Taking $S_{g}=\max$ implies $S$ does not change. Yet since any dissipation heats up the grains, increases $S$, this simply never happens.

\subsection{Tapping: thermal and athermal}

Tapping is accepted as the one experiment naturally explained by ASM [10]. We therefore scrutinize it more closely. A heap of grains has many stable configurations, many local maxima of $S$. The logarithm of this number, typically an increasing function of the density, is the Edward entropy, $S_{E d}(\rho)$. Assuming it is $S_{E d}(\rho)$ that is maximal in equilibrium implies that the density increases under tapping, simply because this increases $S_{E d}$.

This consideration is justified only if $S$ is independent of the density, if the number of microscopic energy states remains the same when the density increases - a proposition that seems unlikely to be true. We note in passing that since $S_{g}$ counts all granular states, in and off equilibrium, including the much more numerous ones with grains flying and jiggling, it is much larger than $S_{E d}$, or

$$
S_{E d} \ll S_{g} \ll S .
$$

The alternative explanation employing GSH involves $S$. In a free column of grains, every layer (carrying the same load from above) has a given pressure. In GSH, with $\pi_{i j}=\pi_{i j}\left(\rho, u_{k l}\right)$, the pressure $P \equiv \frac{1}{3} \pi_{\ell \ell}$ also depends on $\rho, u_{k l}$. Under tapping, because grains periodically lose contact with one another, their deformation is slowly lost, $u_{i j} \rightarrow 0$. As $u_{i j}$ relaxes, $\rho$ increases to maintain the pressure $P$. This is why the density increases, and it is connected to an increase of $S:$ As $u_{i j} \rightarrow 0$, the associated elastic energy dissipates into heat, increasing $S$. This increase is much, much larger than any changes in $S_{E d}$.

It is not hard to discriminate between both explanations experimentally, because the consideration involving $S_{E d}(\rho)$ is independent of the pressure, while in GSH, it results directly from holding the pressure (or stress) constant. If grains are submerged in a liquid of the same density, gravitation does not produce any pressure. Starting from any stress, tapping will diminish $u_{i j}$, and with it the stress, but will not increase the density. Compaction happens then only if an external pressure is applied.

\subsection{The concerns of the athermal community}

A large fraction of the granular physics community believes granular media cannot be treated by conventional tools of theoretical physics, because general principles, including energy conservation, thermodynamics and the concept of equilibrium, are invalid. As should be obvious by now, these are consequences of the athermal model, not abnormalities of granular media as such.

Then there are those who do accept that equilibrium is given by $S=\max$, but take this as a result of equilibrium statistical mechanics alone, lacking any relevance off-equilibria. This is a partial and erroneous view. First of all, the entropy is well defined off-equilibrium, if the system is in local equilibrium, or in generalized equilibrium, in which a few slowly relaxing variables are off their equilibrium values. And it grows continually as the system moves toward global equilibrium. This evolution is accounted for by dissipative terms, which are derived by requiring that $S$ always increases - as we did for the pendulum above, and as was done setting up GSH.

\section{Granular Thermodynamics}

\subsection{Two versions of granular thermodynamics}

The basic thermodynamic relation for a "mechanically stable agglomerate of infinitely rigid grains at rest" is (with $V$ the volume) widely taken as [2,3]

$$
\mathrm{d} V=\left(\mathrm{d} V / \mathrm{d} S_{E d}\right) \mathrm{d} S_{E d} \equiv X \mathrm{~d} S_{E d} .
$$

This ansatz is best appreciated by taking $S_{g}$ as a function of the energy $E$ and volume $V: S_{g}=S_{g}(E, V)$, or

$$
\mathrm{d} S_{g}=\frac{\partial S_{g}}{\partial E} \mathrm{~d} E+\frac{\partial S_{g}}{\partial V} \mathrm{~d} V \equiv \frac{1}{T_{g}} \mathrm{~d} E+\frac{P}{T_{g}} \mathrm{~d} V .
$$

For infinitely rigid grains at rest, as there is no kinetic or deformation energy, we have $E \equiv 0$ - the energy is zero however the grains are arranged. This implies $\mathrm{d} S_{g}=\left(P / T_{g}\right) \mathrm{d} V$, or $\mathrm{d} V=\left(T_{g} / P\right) \mathrm{d} S_{g} \equiv X \mathrm{~d} S_{g}$. Then, clearly, $S_{g}$ reduces to $S_{E D}$.

Because grains are elastic and frequently in motion, the energy does not usually vanish. Hence Eq.(3) holds and is usually written as $\mathrm{d} E=T_{g} \mathrm{~d} S_{g}-P \mathrm{~d} V$. Including microscopic degrees of freedom, we have $E\left(S, S_{g}, V\right)$, or

$$
\mathrm{d} E=T \mathrm{~d} S+T_{g} \mathrm{~d} S_{g}-P \mathrm{~d} V
$$

Next, as the change of the energy with volume depends on the form change, the variable $V$ has to be replaced by $u_{i j}$ and $\rho$, and the description has to be local: Hence we take an energy density $w=w\left(s, s_{g}, \rho, u_{i j}\right)$, depending on the entropy density $s$, reduced entropy density $s_{g}$, or

$$
\mathrm{d} w=T \mathrm{~d} s+T_{g} \mathrm{~d} s_{g}+\mu \mathrm{d} \rho-\pi_{i j} \mathrm{~d} u_{i j}
$$

This is the thermodynamic starting point for GSH $[7,8]$.

\subsection{More on the granular temperature $T_{q}$}

In any uniform medium such as water or air, there are two length scales, macro- and microscopic. All degrees of freedom may be divided into either of these two groups. A macroscopic theory takes those from the first as explicit variables, each with an equation of motion. These including mass, momentum and energy density. Those from the second group are treated summarily, with their contribution to the energy lumped together as heat, and characterized by the temperature $T$. Irreversibility is caused by the macroscopic energy decaying into heat.

In granular media, there is an intermediate group of degrees - momentum and deformation of individual grains. 
In DEM, these are explicit variables. For a macroscopic theory, a summary inclusion again suffices, with their energy lumped into granular heat, quantified by $T_{g}$. That macroscopic energy decays into granular heat, then on to true heat, is what we termed two-stage irreversibilty.

We separate $T_{g}$ from $T$, instead of lumping them into one temperature, not only because of the different length scales. Equally important is the fact that $T_{g}$ is an independent state variable, on which granular dynamics critically depends: The elastic stress relaxes when grains jiggle, when $T_{g} \neq 0$, while it is (within limits) independent of $T$. Lumping both into one temperature obscures this difference. Extending this argument, we see that a third temperature is superfluous. For instance, it is not useful to introduce a temperature for those degrees characterizing the surface roughness of grains. Though the length scale is distinctly smaller, no aspect of the macroscopic granular dynamics depends on an associated temperature. These degrees are part of $T_{g}$. Similarly, it is not useful to introduce a configuration temperature independent from $T_{g}$.

Both $T_{g}$ and $T$ are genuine temperatures, as each characterizes the energy of a group of degrees of freedom. Same holds for the granular and true entropy, $S_{g}$ and $S$ : Each is the logarithm of the number of states in the associated group. Any difficulties treating $T_{g}$ as a temperature arises only because $T$ is ignored. For instance, the granular temperatures $T_{g}$ of two systems in contact are typically different - seemingly a failure of $T_{g}$ to equilibrate. Given two granular systems, 1 and 2, with only 1 being excited, there are, in the steady state, four generally unequal temperatures: $T^{1}, T_{g}^{1}, T^{2}, T_{g}^{2}$. And there are three ongoing energy fluxes: $\left(T_{g}^{1} \rightarrow T^{1}\right),\left(T_{g}^{1} \rightarrow T_{g}^{2}\right),\left(T_{g}^{2} \rightarrow T^{2}\right)$. This is in complete analogy to four conventional thermal systems, (1, 1a, 2, 2a), with only (1a) being heated by an external source, and (1a,1), (1a, 2a), (2a, 2) in pairwise thermal contact. We expect all four temperatures to be different.

Taking the energy density as a function of the two entropy densities, $w=w\left(s, s_{g}\right)$, the conjugate variables are:

$$
T \equiv \partial w / \partial s, \quad T_{g} \equiv \partial w / \partial s_{g}
$$

Denoting $s_{t o t}=s+s_{g}$, we may also write

$$
\mathrm{d} w=T \mathrm{~d} s+T_{g} \mathrm{~d} s_{g}=T \mathrm{~d} s_{t o t}+\left(T_{g}-T\right) \mathrm{d} s_{g},
$$

and identify $T \mathrm{~d} s_{\text {tot }}$ as the equilibrium energy change for changes of the total entropy, and $\left(T_{g}-T\right) \mathrm{d} s_{g}$ as the extra energy contribution for $T_{g} \neq T$. Since $\left(T_{g}-T\right)$ characterizes the non-optimal energy distribution between the granular and microscopic degrees of freedom, the energy $w$ has a minimum at $T_{g}=T$, and is, if expanded, given as: $w \propto\left(T_{g}-T\right)^{2}$. This implies $T_{g}-T$ relaxes until it vanishes.

Now, with $s \gg s_{g}$, and any granular motion at all occurs at $T_{g} \gg T$. Therefore, $T_{g}-T \approx T_{g}, s_{t o t} \approx s$, and the rewriting of Eq.(7) does not change anything. And we may simply take $w \propto T_{g}^{2}$, with $T_{g}$ relaxing until $T_{g}=0$.

Since the granular heat $\int T_{g} \mathrm{~d} s_{g} \propto T_{g}^{2}$ denotes the elastic and kinetic energy of the grains, it reduces, in the limit of vanishing $\rho$, in which only the kinetic part remains, to the energy or temperature of the kinetic theory $T_{k}$, or

$$
T_{k} \propto T_{g}^{2}
$$

A division into three scales works when they are well separated - though this is a problem of accuracy, not viability. Scale separation is well satisfied in largescaled, engineering-type experiments, less so in smallscaled ones. Using glass or steel beads (typically larger) aggravates the problem. Nevertheless, when the system is too small for spatial averaging, one may still average over time and runs to get rid of the fluctuations not contained in a hydrodynamic theory.

\section{References}

[1] D. Bi, S. Henkes, K.E. Daniels, B. Chakraborty. Ann. Rev. Condensed Matter Physics 6: 63-83 (2015);

[2] S.F. Edwards, R.B.S. Oakeshott, Physica A157, 1080 (1989);

[3] R. Blumenfeld, J.F. Jordan, S.F. Edwards. Phys. Rev. Lett. 109, 238001 (2012)

[4] Y.M. Jiang and M. Liu. Eur. Phys. J. E 38: 15 (2015)

[5] D. Kolymbas. Introduction to hypoplasticity, Balkema, Rotterdam, 2000

[6] D.L. Henann and K. Kamrin. Proceedings of the National Academy of Sciences, 110, 6730 (2012).

[7] Y.M. Jiang, M. Liu. Granular Matter, 11:139 (2009). Free copy:www.springerlink.com/content/a8016874j8868u8r/fulltext

[8] Y.M. Jiang and M. Liu. Acta Mech. 225, 2363-2384 (2014)

[9] Y.M. Jiang and M. Liu. Why granular media are thermal, and quite normal, after all. Eur. Phys. J. E (2017) 40: 10

[10] P. Richard, M. Nicodemi, R. Delannay, P. Ribiere, D. Bideau, Nature, 4, 121 (2005) 